\title{
The Effect of Curcumin on Anxiety and Recognition Memory in Kainate Model of Epileptic Rats
}

\author{
H. Y. YOW ${ }^{1}$, N. AHMAD, N. AZMI AND M. MAKMOR-BAKRY*
}

Faculty of Pharmacy, Universiti Kebangsaan Malaysia, Jalan Raja Muda Abdul Aziz, Kuala Lumpur-50300, ${ }^{1}$ School of Pharmacy, Taylor's University (Lakeside Campus), Jalan Taylor's, Subang Jaya-47500, Malaysia

\section{Yow, et al.: Anxiety and Recognition Memory Alteration by Curcumin}

\begin{abstract}
The effect of curcumin had been studied on anxiety and recognition memory in kainic acid-induced epilepsy. A single dose of intraperitoneal kainic acid $(10 \mathrm{mg} / \mathrm{kg})$ was used to induce status epilepticus in female Wistar rats, followed by respective treatments (vehicle dimethyl sulfoxide $50 \%$, curcumin $100 \mathrm{mg} / \mathrm{kg} / \mathrm{d}$ or levetiracetam 100 $\mathrm{mg} / \mathrm{kg} / \mathrm{d}$ ) on the next day for $7 \mathrm{~d}$, with minimum of six rats per group. The behavioural tests were performed before seizure induction and after treatment. Vehicle-treated epileptic rats showed an increase in anxiety-like behaviours in the open field test, which less observed in the light/dark box test. Levetiracetam-treated epileptic rats exhibited anxiolytic behaviours in both the tests. Curcumin-treated epileptic rats exhibited not much difference in anxiety-like behaviour before and after the treatment in open field test, but exhibited anxiolytic behaviours in light/dark box test. Kainic acid impaired the both spatial and non-spatial recognition memories of the rats. Curcumin treatment showed reversal in the non-spatial recognition impaired by kainic acid. In conclusion, kainic acid increases anxiety-like behaviours and impaired recognition memories during the early phase of epileptogenesis and curcumin was potentially improved anxiety and non-spatial recognition memory impaired by kainic acid. These benefits highlighted the potential effect of curcumin to improve psychiatric disorders in epilepsy.
\end{abstract}

Key words: Kainic acid, epileptogenesis, anxiety, cognitive function, novel object recognition, recognition memory

Epilepsy is frequently associated with psychiatric disorders. The prevalence of psychiatric disorders among epilepsy patients is higher compared with the general population, which include depression, anxiety and cognitive dysfunction ${ }^{[1]}$. The underlying psychopathology in epilepsy is unclear. It may be related to the effects of seizure activity or post-ictal state $^{[2]}$. Hence, animal models are utilised to understand the neuropsychological mechanisms in epilepsy ${ }^{[3,4]}$. The use of chemoconvulsant model provides an insight into potential mechanisms of seizure-induced cognitive damage, as well as investigating the effects of therapeutic candidates ${ }^{[5]}$. Seizure induction by chemoconvulsant kainic acid (KA) has been used as a model for temporal lobe epilepsy. KA is preferable in view of its involvement of limbic structures with unique temporal pattern relevance to human temporal lobe epilepsy ${ }^{[6-8]}$. Both systemic and intracerebral administration of KA produce similar brain lesions although systemic administration involves a more widespread of damage ${ }^{[9]}$. However,

*Address for correspondence

E-mail: mohdclinpharm@ukm.edu.my

March-April 2017 systemic administration of $\mathrm{KA}$ is more advantageous over intracerebral administration due to no surgical procedures required, less labour intensive and less time consumed $^{[9]}$.

Curcumin is the main active constituent extracted from rhizome of the plant Curcuma longa L. (Zingiberaceae). Recent preclinical studies demonstrated that curcumin may exhibit promising anticonvulsant $\operatorname{effect}^{[10,11]}$ as well as potential antiepileptogenic effect ${ }^{[12,13]}$. It also has been shown to possess protective effects in ameliorating cognitive impairments and anxiety in epileptic animals ${ }^{[11,13,14]}$. These benefits highlighted curcumin as a potential antiepileptic agent for clinical management. Although previous studies reported that

This is an open access article distributed under the terms of the Creative Commons Attribution-NonCommercial-ShareAlike 3.0 License, which allows others to remix, tweak, and build upon the work non-commercially, as long as the author is credited and the new creations are licensed under the identical terms

Accepted 16 March 2017

Revised 20 December 2016

Received 09 August 2016

Indian J Pharm Sci 2017;79(2):267-276 
the neuroprotective effects of curcumin on behavioural changes in epileptic animal, different models of epilepsy (mainly pentylenetetrazol-induced epilepsy model) were employed in these studies ${ }^{[11,13,14]}$.

Hence, this study aimed to evaluate the effects of intraperitoneal curcumin on anxiety and recognition memory in KA-induced epilepsy. This study also provides an insight into the behavioural changes during epileptogenesis since previous behavioural studies were mostly focused in chronic epilepsy models.

\section{MATERIALS AND METHODS}

\section{Experimental animals:}

A total of 40 female Wistar rats weighing 200-220 g were kept under controlled environment conditions (50-60\% humidity, $23 \pm 1^{\circ}, 12 \mathrm{~h}$ light/12 h dark cycle) with free access to food and water. The rats were housed in groups of three and allowed to acclimatise for $1 \mathrm{w}$ prior to experiment. All procedures were performed in the light phase. All protocols were approved by Animal Ethics Committee of University (UKMAEC approval number: $\quad$ FF/2013/MOHD/31-JAN./491-MARCH2013-APRIL-2015).

\section{Seizure induction and treatment:}

For status epilepticus (SE) induction, the rats were intraperitoneally injected with a single dose of 10 $\mathrm{mg} / \mathrm{kg}$ KA (Sigma-Aldrich, St. Louis, USA), which dissolved with isotonic saline in $3 \mathrm{ml} / \mathrm{kg}$. In the control group, vehicle (saline) injection was administered intraperitoneally at the same volume as KA-induced group. After the injection, the rats were monitored for behavioural changes for $6 \mathrm{~h}$. Seizure severity was scored according to Racine scale ${ }^{[15]}$ : stage 1-mouth and facial movement; stage 2-head nodding; stage 3-forelimb clonus; stage 4-rearing and stage 5-rearing and falling accompanied by generalised clonic seizures. Rats that achieved stage 4 or stage 5 of SE were randomly distributed to group 2, 3 or 4 . The rats that survived until completion of treatment were included in the behavioural assessment. Each group consisted of a minimum of six animals were as following. Group 1: control group, in which the rats were saline-treated (without seizure induction) and treated with vehicle 50\% dimethyl sulphoxide (DMSO) intraperitoneally; group 2: negative control group, in which the rats were given KA for seizure induction and treated with vehicle $50 \%$ DMSO intraperitoneally; group 3: positive control group, in which the rats were given KA and treated with antiepileptic drug levetiracetam 100 $\mathrm{mg} / \mathrm{kg} / \mathrm{d}$ intraperitoneally; group 4: curcumin treated group, in which the rats were given KA and treated with curcumin $100 \mathrm{mg} / \mathrm{kg} / \mathrm{d}$ intraperitoneally.

The treatment was initiated the day after the seizure induction and terminated after $7 \mathrm{~d}$ of treatment. Curcumin $(\geq 94 \%$ curcuminoid content, $\geq 80 \%$ curcumin; Sigma-Aldrich, St. Louis, USA) was prepared freshly by suspending in 50\% DMSO (Fisher Scientific, Loughborough, UK) and administered at dose of $100 \mathrm{mg} / \mathrm{kg} / \mathrm{d}^{[16,17]}$. Levetiracetam (UCB Pharma, Smyrna, USA) was diluted with normal saline and given at dose $100 \mathrm{mg} / \mathrm{kg} / \mathrm{d}^{[18]}$. The vehicle, DMSO $50 \%$ was given intraperitoneally for groups 1 and 2 at volume of $10 \mathrm{ml} / \mathrm{kg}$.

\section{Behavioural studies:}

All animal were subjected to a serial behavioural test before seizure induction and after the treatment as fig. 1 . The behavioural tests were carried out chronologically from the least to the most aversive, with an inter-test interval of one day. Anxiety tests, which comprised of open field test (OFT) and light/dark box test (LDT) have been carried out first; followed by recognition memory tests, i.e. novel object recognition (NOR) task and object location recognition task in latter experiment. All tests were conducted within $1 \mathrm{w}$. On the day of testing, the animals were placed in the quiet and dark experimental room at least $30 \mathrm{~min}$ before testing. Behavioural test was carried out only if seizure was not observed for at least $30 \mathrm{~min}$ before testing. If a spontaneous seizure developed during testing, the test was halted and the rat was returned to its cage. The testing was repeated at interval of $1 \mathrm{~h}$ after seizure cessation. During the test, the behaviour of rat was recorded by an overhead video camera for subsequent analysis, with presence of personnel to allow evaluation off-line.

OFT was conducted in a square open field of $100 \times 100 \times 50 \mathrm{~cm}$, made of Plexiglas with the inside walls painted with matt grey and a white floor that divided into 25 squares of $20 \times 20 \mathrm{~cm}^{[19]}$. The open field was divided into two zones: outer (i.e. peripheral squares adjacent to the walls) and inner zone. The test was carried out in a dark room and the floor was dimly illuminated (10 lux). The rat was located in the centre of the open field and allowed for $5 \mathrm{~min}$ exploration. After the exploration, the rat was returned into the cage. The open field was cleaned thoroughly with $10 \%$ ethanol and dried with paper towels before each trial to avoid odour trails. The following variables were measured: 
www.ijpsonline.com

\begin{tabular}{|c|c|c|}
\hline Pre-treatment & Treatment & Post-treatment \\
\hline
\end{tabular}
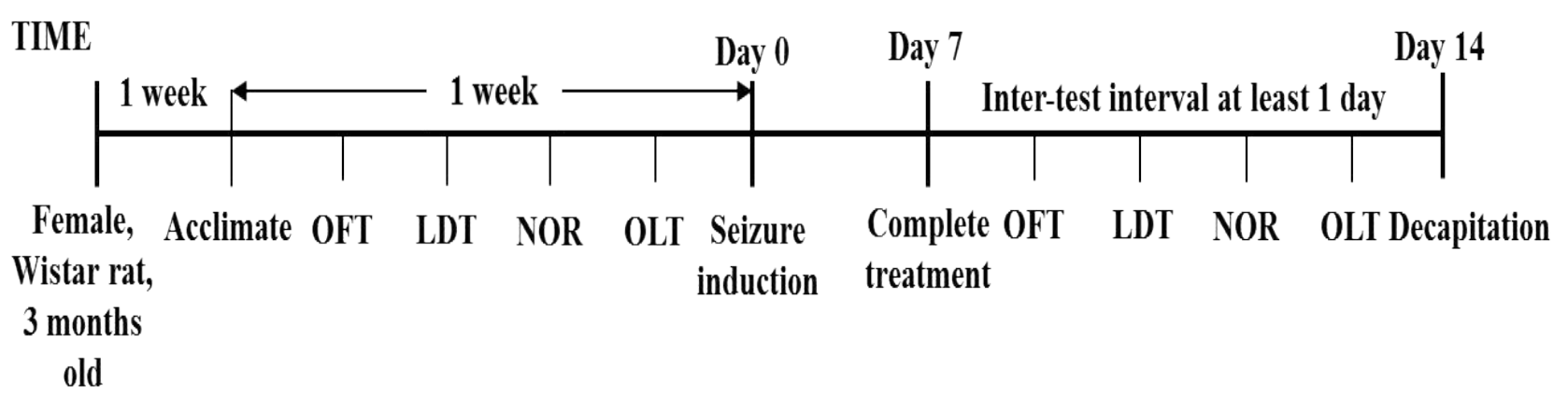

Fig. 1: Schematic behavioural testing schedule

OFT-open field test; LDT-light/dark box test; NOR- novel object recognition; OLT- object location recognition task

the number of inner and outer squares crossed; time spent in each zone, number of rearing, grooming and stretch attend posture; and total number of faecal boli and urine drops ${ }^{[19]}$.

LDT was carried out in a rectangular Plexiglas box sized $27 \times 45 \times 27 \mathrm{~cm}$ with translucent covers that constitute two compartments, i.e., dark and light compartments ${ }^{[19]}$. The dark compartment constituted one third of the box $(27 \times 18 \mathrm{~cm})$, painted black and illuminated by a dim red bulb (4 lux). The other two thirds were light compartment $(27 \times 27 \mathrm{~cm})$ that painted white and lit up with a white bulb (400 lux). The floor in white and black compartments was divided into 9 and 6 of squares sized $9 \times 9 \mathrm{~cm}$, respectively. An opening of $7 \times 7 \mathrm{~cm}$ was located at floor level in the middle of the partition wall between compartments. The test was conducted in a dark room. Rats were individually placed in the centre of the light compartment facing the opening and allowed for 5 min exploration. After the exploration, the rat was transferred into its cage. Each compartment was cleaned thoroughly with $10 \%$ ethanol and dried with paper towels before each trial. The following variables were measured: time spent in each compartment, the number of transitions between compartments, the latency of first entry to each compartment, the number of squares crossed, number of rearing, grooming and stretch attend posture, total number of faecal boli and urine drops. Note that the time spent in the light compartment excluded the initial latency to enter the dark compartment (first visit when rats exposed to the box).

The NOR task is adapted from the protocol of Ennaceur and Delacour ${ }^{[20]}$. The test was performed in a square open box $60 \times 60 \times 40 \mathrm{~cm}$, made of Plexiglas with the inside walls painted with black and a white floor that divided into 16 squares of $15 \times 15 \mathrm{~cm}$. The objects used for discrimination were beverage cans covered with colour papers, glass bottles and rectangular boxes, which were varied in size from $15 \times 15 \times 14 \mathrm{~cm}$ to $7 \times 7 \times 12 \mathrm{~cm}$. The objects were located in two adjacent corners of the arena that approximately $10 \mathrm{~cm}$ from the side walls. The test was carried out in a quiet, dark room and dimly illuminated (50 lux) by a light bulb. Habituation session was carried out for each rat individually in the box for $5 \mathrm{~min}$ on the day before the behavioural test. On the test day, the rats were placed in the dark experimental room at least 30 min before testing. Subsequently, the rats were placed individually into the open field facing away from the objects. The rats were allowed to explore two identical objects (A1, A2) in a sample phase (T1) and then followed by a choice phase (T2), in which they were reintroduced to a familiar object (A3) that is identical to object in the first trial (A1) and a novel object (B) that replaced the familiar object (A2). Each trial lasted for 3 min with interception of $5 \mathrm{~min}$ inter-trial interval (spent in home cage). The boxes and objects were cleaned between each trial using $10 \%$ ethanol to avoid olfactory trails during the habituation session and experiments. The position of objects was arranged in a balanced manner and objects were randomly exchanged to minimise potential place and object preference effects. Exploration was defined as the animal's nose directed to the object at a distance $\leq 2 \mathrm{~cm}$ and/or touching it with the nose. Turning around or sitting on the object was not considered as an exploratory behaviour ${ }^{[20]}$. The basic measure was the total time spent by rats exploring each object (namely A1, A2, A3 and B) in both trials (T1 and T2). Total time spent by rats in exploring both objects during $\mathrm{T} 1$ and $\mathrm{T} 2$ were calculated according to the method of Ennaceur and Delacour ${ }^{[20]}$ using the Eqns., e1 $=\mathrm{A} 1+\mathrm{A} 2$ and $\mathrm{e} 2=\mathrm{A} 3+\mathrm{B}$, respectively. 
For object location recognition task, the apparatus and procedures were similar as NOR task except for the choice phase. In this task, two identical sample objects (A3 and A4) were used in the choice phase, in which object $A 3$ replaced in the same position as the A1 and A4 was placed at the corner adjacent to the original position of A2 (two objects were in diagonal corners). The basic measure was the total time spent by rats exploring each object (namely A1, A2, A3 and A4) in both trials (T1 and T2). Total time spent by rats in exploring both objects during $\mathrm{T} 1$ and $\mathrm{T} 2$ were calculated according to the method of Ennaceur and Delacour ${ }^{[20]}$ using the Eqns., e1 $=\mathrm{A} 1+\mathrm{A} 2$ and $\mathrm{e} 2=$ $\mathrm{A} 3+\mathrm{B}$, respectively.

\section{Statistical analysis:}

Data were analysed using the statistical package for the Social Sciences version 21 (IBM Corporation, New York, US). To compare the difference before and after the experiment, paired t-test was performed within the same experimental group for behavioural tests. For NOR and object location recognition tasks, paired t-test was used to compare within the same experimental group for the time spent in exploring the identical objects (A1, A2) in the sample phase, the time spent in exploring the familiar object (A3) and novel object (B) or novel location (A4) during choice phase or the total exploration time in the sample (e1) versus choice phase (e2). One-way analysis of variance (ANOVA) was used to compare the differences for more than 2 groups.

\section{RESULTS AND DISCUSSION}

KA was administered to 34 rats, out of which 28 $(82.35 \%)$ rats were developed at stage $4(n=8)$ or stage $5(\mathrm{n}=20)$ of SE, which lasting for $169.30 \pm 53.36 \mathrm{~min}$, but only $19(67.86 \%)$ survived until completion of treatment. Those rats, which failed to achieve stage 4 or 5 (stage $1, n=2$; stage $2, n=3$; stage $3, n=1$ ) and died $(n=9)$ were excluded for the subsequent analysis. There was no seizure activity observed in saline-induced control group $(\mathrm{n}=6)$.

In the OFT, there was no significant difference for the total locomotor activity (sum of the number of lines crossed and frequency of rearing) between pre- and post-treatment for all groups. However, the locomotor activity in the inner zone of open field was significantly decreased in non-epileptic controls $(\mathrm{t} 5=2.900, \mathrm{P}=0.034)$ and vehicle-treated epileptic rats $(\mathrm{t} 5=2.513, \mathrm{P}=0.046)$. This result paralleled with the decrease in total number of lines crossed in the inner zone ( $\mathrm{t} 5=2.782$, $\mathrm{P}=0.039$ for saline-DMSO; fig. $2 \mathrm{a}$ ) and time spent in the inner zone $(\mathrm{t} 5=3.137, \mathrm{P}=0.026$ for saline-DMSO; $\mathrm{t}(5)=3.913, \mathrm{P}=0.011$ for KA-DMSO; fig. $2 \mathrm{~b}$ ), which indicate increased anxiety-like behaviours. Although the total number of line crossings in the inner zone for vehicle-treated epileptic rats during the post treatment $(16.00 \pm 3.46)$ was lesser than those during pre-treatment $(30.67 \pm 5.50)$, this difference was not significant. The frequency of rearing in inner zone was significantly reduced in all groups after respective treatment, except for levetiracetam treated group $(\mathrm{t} 5=2.513, \mathrm{P}=0.046$ for saline-DMSO; $\mathrm{t} 5=4.129, \mathrm{P}=0.009$ for KA-DMSO; $\mathrm{t} 5=2.298, \mathrm{P}=0.007$ for KA-curcumin). Levetiracetamtreated epileptic rats had shown an increase in total line crossings in the outer zone ( $\mathrm{t} 6=2.534, \mathrm{P}=0.044)$, but decrease in urination $(\mathrm{t} 6=2.464, \mathrm{P}=0.049)$ and stretch attend postures ( $\mathrm{t} 6=5.528, \mathrm{P}=0.001)$ after the treatment. There was no difference observed in all treated and control groups for other behavioural parameters.

In the LDT, there was no significant difference in total locomotor activity between pre- and post-treatment for all groups. However, the locomotor activity of levetiracetam-treated epileptic rats in light zone was increased after treatment ( $\mathrm{t} 6=2.651, \mathrm{P}=0.038)$. This result was consistent with the increase in time spent in the light zone ( $\mathrm{t} 6=-2.743, \mathrm{P}=0.034$; fig. 3a), total

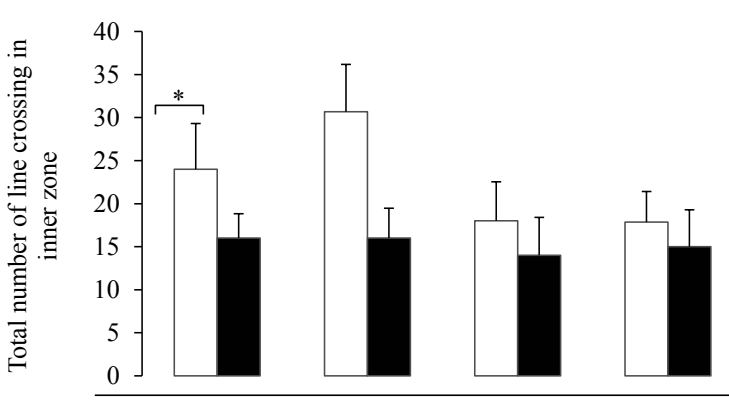

(a)

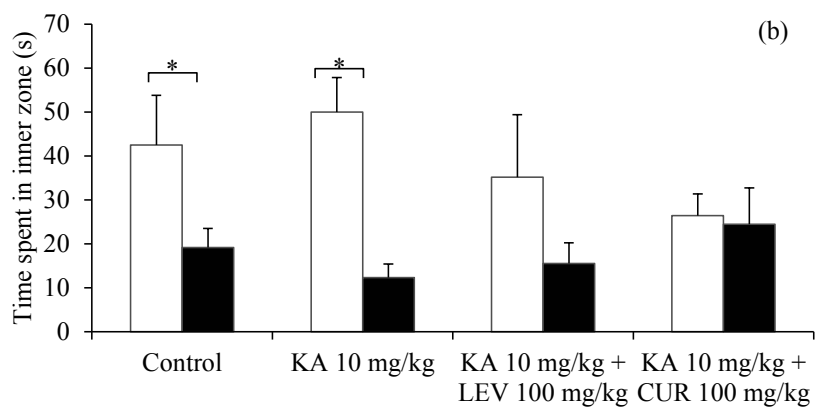

Fig. 2: The behaviour of epileptic and non-epileptic controls in the open field test

(a) Total number of line crossing in inner zone; (b) Time spent in inner zone. $\square$ Pre-treatment; $\square$ post treatment. KA is kainic acid; $L E V$ is levetiracetam and CUR is curcumin 
line crossings in the light zone $(\mathrm{t} 6=-2.677, \mathrm{P}=0.037)$ and number of transitions from dark to light zone ( $\mathrm{t} 6=2.545, \mathrm{P}=0.044$; fig. $3 \mathrm{~b}$ ), indicating a decrease in anxiety level. Contrastingly, the non-epileptic controls demonstrated a decrease in transitions from dark to light zone ( $\mathrm{t} 5=3.051, \mathrm{P}=0.028$; fig. $3 \mathrm{~b}$ ), but yet no significant alteration in neither time spent in the light zone nor locomotion in the light zone. Curcumin-treated epileptic rats demonstrated a significant decrease in stretch attends $(\mathrm{t} 5=4.824, \mathrm{P}=0.005)$ and urination ( $\mathrm{t} 5=3.105, \mathrm{P}=0.027)$ after the treatment. There was no change observed in all treated and control groups for latency to enter each zone, defecation, rearing and grooming.

In the NOR task, the rats from all treatment groups spent similar time in exploring the identical objects, A1 and A2 during the sample phase before and after treatment (Table 1). Before any treatment, the rats were able to discriminate the novel object (B) from the familiar object (A3) during the choice phase in all groups ( $\mathrm{t} 5=$ $2.561, \mathrm{P}=0.043$ for saline-DMSO; $\mathrm{t} 5=-3.991, \mathrm{P}=0.010$ for KA-DMSO; $\mathrm{t} 6=-4.625, \mathrm{P}=0.004$ for KA-LEV; $\mathrm{t} 5=5.537, \mathrm{P}=0.003$ for KA-CUR; fig. 4a). After the treatment, only non-epileptic controls and curcumintreated epileptic rats significantly discriminated the novel object from the familiar object $(\mathrm{t} 5=4.774$, $\mathrm{P}=0.005$ for saline-DMSO; $\mathrm{t} 5=3.186, \mathrm{P}=0.024$ for KA-CUR; fig. 4b). There was no significant difference
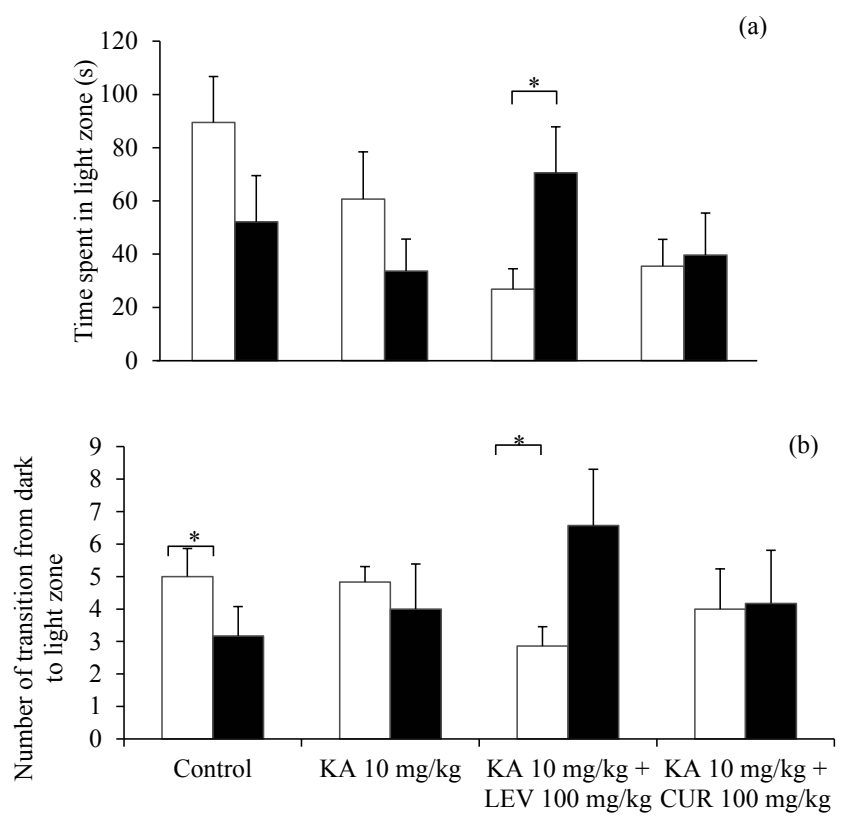

Fig. 3: The behaviour of epileptic and non-epileptic controls in the light/dark box test

(a) The time spent in the light zone; (b) the number of transitions from dark to light zone. $\square$ Pre-treatment; $\square$ post treatment. KA is kainic acid; LEV is levetiracetam and CUR is curcumin
TABLE 1: EXPLORATORY ACTIVITIES OF THE EPILEPTIC AND NON-EPILEPTIC CONTROLS IN THE SAMPLE PHASE OF THE NOVEL OBJECT RECOGNITION TASK

\begin{tabular}{lcccc}
\hline Group & \multicolumn{2}{c}{ Pre-treatment } & \multicolumn{2}{c}{ Post-treatment } \\
\cline { 2 - 5 } & Object A1 & Object A2 & Object A1 & Object A2 \\
\hline Control & $22.50 \pm 1.77$ & $17.83 \pm 3.91$ & $11.67 \pm 2.50$ & $13.17 \pm 1.47$ \\
KA & $13.00 \pm 0.89$ & $13.17 \pm 1.96$ & $8.17 \pm 1.92$ & $11.50 \pm 3.44$ \\
KA+LEV & $11.43 \pm 2.38$ & $11.00 \pm 2.33$ & $18.29 \pm 3.94$ & $11.86 \pm 2.36$ \\
KA+CUR & $18.83 \pm 2.59$ & $18.5 \pm 2.92$ & $9.33 \pm 2.03$ & $10.33 \pm 1.45$ \\
\hline
\end{tabular}

Rats did not demonstrate any significant difference in the exploration time of identical objects, A1 and A2 before seizure induction (pre-treatment) and after the treatment (post-treatment). Data expressed as mean in seconds $( \pm S E M) ; n=6-7$ animals per group. KA: kainic acid $(10 \mathrm{mg} / \mathrm{kg})$; LEV: levetiracetam (100 mg/kg); CUR: curcumin $(100 \mathrm{mg} / \mathrm{kg})$
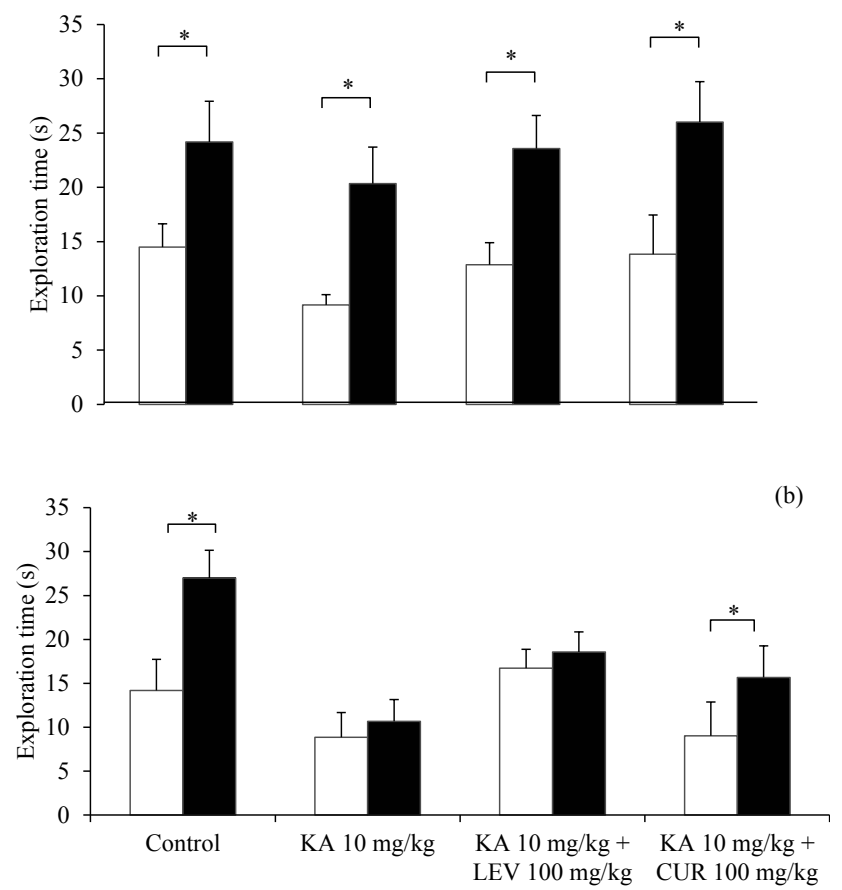

Fig. 4: Effect of levetiracetam and curcumin treatment on epileptic rats in novel object recognition task

Recognition task during choice phase of (a) pre-treatment and (b) post-treatment. $\square$ Object A3; $\square$ Object B. KA is kainic acid; LEV is levetiracetam and CUR is curcumin

in total exploratory activities during the sample phase (e1) and choice phase (e2) within the group comparison.

Similar to the NOR task, the rats from all treatment groups spent similar time in exploring the identical objects, A1 and A2 during the sample phase before and after treatment in object location recognition task as depicted in Table 2. Before any treatment, the rats recognised the identical object that had been changed in location in all groups as illustrated in fig. $5 \mathrm{a}(\mathrm{t} 5=$ 3.724, $\mathrm{P}=0.014$ for saline-DMSO; $\mathrm{t} 5=-2.561, \mathrm{P}=0.043$ for $\mathrm{KA}-\mathrm{DMSO}$; $\mathrm{t} 6=-5.695, \mathrm{P}=0.001$ for $\mathrm{KA}-\mathrm{LEV}$; $\mathrm{t} 5=-2.298, \mathrm{P}=0.033$ for KA-CUR). However, after the 
TABLE 2: EXPLORATORY ACTIVITIES OF THE EPILEPTIC AND NON-EPILEPTIC CONTROLS IN THE SAMPLE PHASE OF THE OBJECT LOCATION RECOGNITION TASK

\begin{tabular}{lcccc}
\hline Group & \multicolumn{2}{c}{ Pre-treatment } & \multicolumn{2}{c}{ Post-treatment } \\
\cline { 2 - 5 } & Object A1 & Object A2 & Object A1 & Object A2 \\
\hline Control & $11.17 \pm 2.02$ & $16.17 \pm 3.20$ & $12.50 \pm 0.85$ & $16.67 \pm 2.28$ \\
KA & $11.17 \pm 1.45$ & $9.83 \pm 1.19$ & $15.33 \pm 3.30$ & $9.33 \pm 2.49$ \\
KA+LEV & $11.71 \pm 0.71$ & $12.71 \pm 2.17$ & $12.29 \pm 1.80$ & $17.57 \pm 2.23$ \\
KA+CUR & $4.83 \pm 1.68$ & $10.00 \pm 2.18$ & $10.83 \pm 1.35$ & $11.67 \pm 1.91$ \\
\hline
\end{tabular}

Rats did not demonstrate any significant difference in exploration time of identical objects, A1 and A2 before seizure induction (pre-treatment) and after the treatment (post-treatment). Data expressed as mean in seconds $( \pm S E M)$; $n=6-7$ animals per group. KA: kainic acid $(10 \mathrm{mg} / \mathrm{kg})$; LEV: levetiracetam (100 mg/kg); CUR: curcumin $(100 \mathrm{mg} / \mathrm{kg})$

seizure induction and treatment, only non-epileptic controls discriminated the novel location from the familiar location $(\mathrm{t} 5=2.298, \mathrm{P}=0.033$ for saline-DMSO; fig. 5b). The total exploratory activities during sample phase (e1) and choice phase (e2) is no different within the group comparison.

Several test paradigms have been developed to assess anxiety and cognitive function in animals. Unconditioned tests are the most frequently used paradigms because they are thought to be manifested generalised anxiety symptoms in human ${ }^{[21]}$. The tests include OFT, LDT, elevated plus maze and hole board, which require no training and animals usually confronted with a novel environment or stimulus directly ${ }^{[21]}$. Hence, OFT and LDT were performed to evaluate the anxiety behaviour. Object recognition task, also known as the NOR task is a commonly used test for declarative memory, which depends on the rodents' innate explorative behaviour of novel objects ${ }^{[20]}$. The NOR task has become the test of choice since it requires no rule of learning or food reinforcement and only little pre-training or habituation required ${ }^{[22]}$. Spatial learning and memory test are commonly assessed with a battery of tests such as radial arm maze, Morris water maze and object location recognition task ${ }^{[23]}$. However, the use of Morris water maze or radial arm maze could probably introduce stress on epileptic rats since these tasks involve aversive methods such as water and food deprivation $^{[24]}$. Therefore, the current study evaluated spatial learning and memory performance by using object location recognition task, which is less stressful and no rule of learning or rewards required ${ }^{[23]}$.

Levetiracetam had been shown to act uniquely on a specific binding site, synaptic vesicle protein $2 \mathrm{~A}$ (SV2A) in addition to its anticonvulsant mechanisms on calcium channels and $\mathrm{GABA}_{\mathrm{A}}$ receptors ${ }^{[25]}$. It was
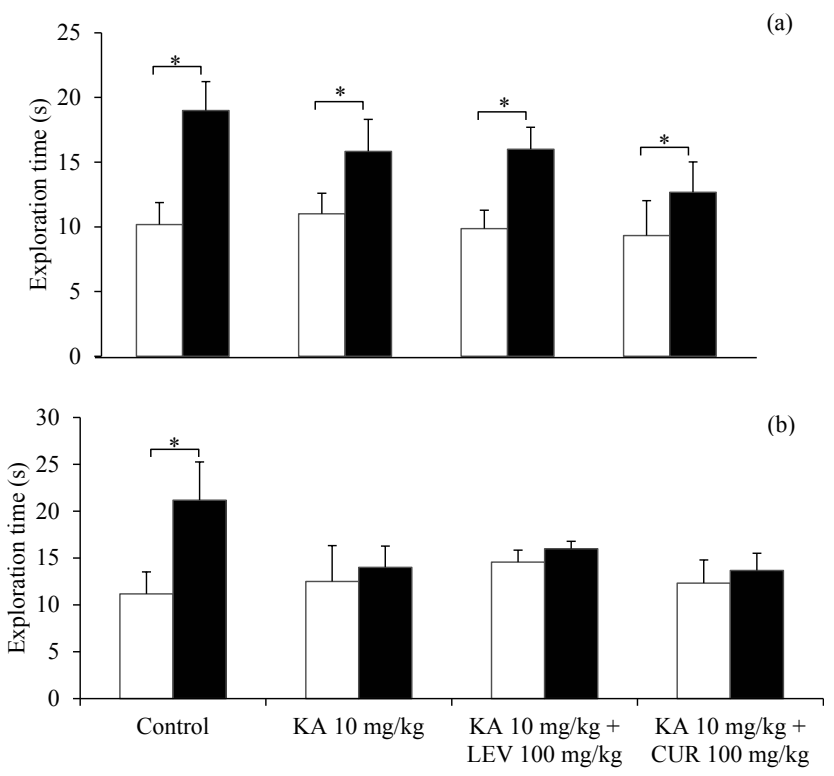

Fig. 5: Effect of levetiracetam and curcumin treatment on epileptic rats in object location recognition task

Recognition task during choice phase of (a) pre-treatment and (b) post-treatment. $\square$ Object A3; $\square$ object $A 4$. KA is kainic acid; LEV is levetiracetam and CUR is curcumin

selected as a positive control group in this study because of its potential anxiolytic activity either in animals ${ }^{[26]}$ or humans ${ }^{[27]}$. A dose of $100 \mathrm{mg} / \mathrm{kg}$ levetiracetam had been demonstrated to have antiepileptogenic effect ${ }^{[18]}$. Therefore, this dosage was selected to investigate whether it could modify the behavioural alterations after seizure induction. Similarly, curcumin with dose of $100 \mathrm{mg} / \mathrm{kg}$ was selected in view of its significant antiepileptic effects at this dosage $\mathrm{e}^{[16,17]}$.

An overview of the behavioural alterations between groups was summarised in Table 3. The control group showed markedly reduced locomotion and time spent in inner zone during OFT, as well as reduced frequency of transitions between compartments in LDT. These parameters indicated an increase of anxiety in control group, which might be related to the nature of the rats to novel compartment and central area ${ }^{[28]}$. Although previous studies ${ }^{[29,30]}$ have shown the effect of DMSO on behaviours, the effect of curcumin on behaviours can be defined from the effect of DMSO with the DMSO treated only control group. For vehicle DMSO treated epileptic rats, the anxiety level was increased in the OFT with reduced inner zone locomotion and time spent in inner zone, whereas lack of anxiety-like behaviours observed in the LDT. This inconsistency might be due to variation between tests on aspects of anxiety or intra-individual fluctuations in anxiety ${ }^{[31]}$. Therefore, the possible anxiety could be undetectable in the LDT among vehicle-treated epileptic rats. 
TABLE 3: COMPARISON OF BEHAVIOURAL ALTERATIONS IN THE LEVETIRACETAM- AND CURCUMINTREATED EPILEPTIC RATS

\begin{tabular}{|c|c|c|c|c|}
\hline \multirow[b]{2}{*}{ Test } & \multicolumn{4}{|c|}{ Behavioural alterations } \\
\hline & Locomotor activity & Exploratory activity & Anxiety-like behaviour & $\begin{array}{l}\text { Recognition } \\
\text { performance }\end{array}$ \\
\hline \multicolumn{5}{|c|}{ Open field test } \\
\hline Control & Decreased (inner zone) & Decreased rearing (inner zone) & Decreased time spent in inner zone & - \\
\hline KA & Decreased (inner zone) & Decreased rearing (inner zone) & Decreased time spent in inner zone & - \\
\hline KA+LEV & Normal & Normal & $\begin{array}{c}\text { Decreased stretch attend postures } \\
\text { and urination }\end{array}$ & - \\
\hline $\mathrm{KA}+\mathrm{CUR}$ & Normal & Decreased rearing (inner zone) & Normal & - \\
\hline \multicolumn{5}{|c|}{ Light/dark box test } \\
\hline Control & Normal & Normal & Decreased frequency of transitions & - \\
\hline KA & Normal & Normal & Normal & - \\
\hline KA+LEV & Increased (light zone) & Normal & $\begin{array}{l}\text { Increased time spent in light zone, } \\
\text { frequency of transitions }\end{array}$ & - \\
\hline $\mathrm{KA}+\mathrm{CUR}$ & Normal & Normal & $\begin{array}{c}\text { Decreased stretch attend postures } \\
\text { and urination }\end{array}$ & - \\
\hline \multicolumn{5}{|c|}{ Novel object recognition task } \\
\hline Control & - & Normal & - & Normal \\
\hline KA & - & Normal & - & Impaired \\
\hline KA+LEV & - & Normal & - & Impaired \\
\hline $\mathrm{KA}+\mathrm{CUR}$ & - & Normal & - & Normal \\
\hline \multicolumn{5}{|c|}{ Object location recognition task } \\
\hline Control & - & Normal & - & Normal \\
\hline KA & - & Normal & - & Impaired \\
\hline KA+LEV & - & Normal & - & Impaired \\
\hline $\mathrm{KA}+\mathrm{CUR}$ & - & Normal & - & Impaired \\
\hline
\end{tabular}

KA: kainic acid (10 mg/kg); LEV: levetiracetam (100 mg/kg); CUR: curcumin $(100 \mathrm{mg} / \mathrm{kg})$

Since these anxiety tests assess on different aspects of anxiety, the present data suggested that the anxiety level was increased in epileptic rats, as reported in previous studies $^{[3,32]}$.

However, in the kainate model of epilepsy, epileptic rats were previously reported to have decreased anxiety-like behaviours compared to controls ${ }^{[33,34]}$. These behavioural alterations could be a reflection of disinhibited hyperactive behaviour, which might be related to the collateral brain lesions in ventral hippocampus, entorhinal and amygdala (i.e. fear expression networks ${ }^{[33,34]}$. Damage on these networks potentially cause an inaccurate interpretation on threatening situations and thus, causing a reduction in anxiety or enhance the impulsive inadapted behaviour $^{[33]}$. The discrepancy of current result could be related to the time for behavioural testing. Studies showed that epileptic rats spent a prolonged period, approximately $5 \mathrm{mo}$ to reach a maximal seizure frequency, i.e., plateau phase after seizure induction ${ }^{[35]}$. Therefore, studies on epilepsy at time point less than 5 mo may generally reveal less severe behavioural deficits due to less frequent spontaneous seizures and less neuropathological changes involved ${ }^{[33]}$. In this study, the behavioural testing was performed approximately one week post SE, hence, the result was parallel to studies on epileptic animal at time point of one to three months post $\mathrm{SE}^{[3,32]}$. However, the association between seizure-related anxiety and the duration of the epileptic state remains unclear. A recent study revealed that the duration of epilepsy did not affect the spatial memory impairment in epileptic animals ${ }^{[36]}$ and cognitive deficits occurred as early as after SE, at the time point before chronic epilepsy begins ${ }^{[24]}$. In addition, other factors such as animal factor (e.g. age, strain and sex), treatment protocol (e.g. use of antiepileptic agent to stop the SE and method of seizure induction) and experimental conditions might contribute to the result discrepancy between studies.

Levetiracetam-treated epileptic rats exhibited anxiolytic behaviours in both OFT and LDT. The levetiracetamtreated epileptic rats showed decreased stretch attends and urination in the OFT. These behavioural alterations might be indicated levetiracetam potentially exhibited an anxiolytic effect. Stretch attend posture is a risk assessment behaviour when facing a threatening stimulus, indicating the animal are hesitant to move ${ }^{[21]}$. Defecation and urination are other indices of anxiety, 
which are controlled by autonomic nervous system ${ }^{[37]}$. Hence, decreased stretch attends and urination might indicate a lower level of anxiety. Consistently, in LDT, levetiracetam treatment increased both locomotion and time spent in light compartment, as well as increased frequency of transitions and reduced stretch attends in epileptic rats. All these behavioural alterations indicated levetiracetam has potential anxiolytic effects in epileptic rodents. The result in the present study reconfirmed the anxiolytic effect of levetiracetam.

Curcumin-treated epileptic rats did not demonstrate anxiety-like behaviours before and after the treatment in OFT. As compared to non-epileptic control and vehicle-treated epileptic group, curcumin treatment might have some anxiolytic effects in ameliorating the anxiety existence either in controls or epileptic rats. In LDT, curcumin-treated epileptic rats exhibited a decrease in stretch attend postures and urination. These behavioural changes indicated anxiolytic-like effects. Taken together, these changes suggested that curcumin has a potential anxiolytic action although much less than levetiracetam. In fact, a previous study also reported that curcumin demonstrated potential protective effects in improving anxiety in animals ${ }^{[14]}$.

In our current study, KA-induced SE not only increases anxiety-like behaviours, but also impaired both spatial and non-spatial recognition memory in rodents. KA had been demonstrated to impair animals' recognition memory in an object recognition task ${ }^{[24]}$. However, a study showed that pilocarpine, another chemoconvulsant post-SE model, induced epilepsy in rats but did not impair recognition memory ${ }^{[34]}$. This discrepancy in finding is explainable by the use of different experimental models of epilepsy that produce different patterns of brain lesions ${ }^{[7]}$ and the use of diazepam to stop the SE might mask the real behavioural deficits that may have occurred. The mechanism of these behavioural changes remains unclear. It is possible that the mechanism is attributable to hippocampal neuronal cell death induced by $\mathrm{KA}^{[33,38]}$. Hippocampus plays a critical role in anxiety ${ }^{[39]}$ and spatial cognition ${ }^{[40]}$. In view of the involvement in non-spatial cognition deficits, it is possible that KA also causes damage in perirhinal cortex in addition to hippocampal regions.

Our findings showed that levetiracetam did not reverse cognitive impairments induced by KA, neither in spatial nor in non-spatial recognition memory. It could be beneficial if newer antiepileptic agents are not only improving the symptoms of epilepsy, but also improve cognitive function in patients. However, levetiracetam, a new generation antiepileptic agent, failed to alter cognitive performance ${ }^{[41,42]}$. It was not until recent, patients with epilepsy were demonstrated to exhibit positive effects on cognitive function following levetiracetam therapy ${ }^{[43]}$. In addition, chronic levetiracetam treatment ( $4 \mathrm{w})$ had been shown to improve non-spatial recognition in Alzheimer's disease animal model ${ }^{[4]}$. Nevertheless, this improvement was not observed in acute treatment. It is suggested that duration of treatment and experimental design play a critical role in the effects of levetiracetam on memory. Similar to the finding by Zhou et al. ${ }^{[45]}$, the levetiracetam-treated group did not restore impaired spatial memory induced by KA in the present study.

Curcumin exerted potential effects in ameliorating anxiety and restoring non-spatial recognition memory impairment induced by KA although no effect on spatial recognition impairment was demonstrated. In the NOR task, only curcumin-treated animals showed intact non-spatial recognition memory comparable to the control group, similar to findings from other studies $^{[11,13,14]}$. Curcumin, however, did not reverse the impaired spatial memory unlike other studies, which have shown that curcumin ameliorated SE-induced spatial memory impairment ${ }^{[12,46]}$. It is debatable among researchers that these discrepancies might be due to different models of epilepsy and different treatment protocols of curcumin employed. In addition, sex of the rats might be attributed to this contradicted finding. In spatial memory task, male rats demonstrated better performance than female rats particularly when oestrogen and progesterone levels are highest in the oestrous cycle ${ }^{[47]}$. Hence, the oestrous cycle phase in female rats might influence the result of object location recognition task in this study.

Behavioural alterations in kainate-induced SE model occurred during the early phase of epileptogenesis. Levetiracetam exhibited anxiolytic effects without improvement of cognitive impairments caused by $\mathrm{KA}$; whereas, curcumin potentially improved anxiety and non-spatial recognition impairment in this chemoconvulsant model of epilepsy. These benefits demonstrated the potential effect of curcumin in improving psychiatric-related disorders in addition to control epilepsy in clinical management. The findings in the present works, together with the previous reports, provide a collective knowledge on effects of curcumin on behavioural changes, particularly in KA- 
induced behavioural changes. In addition, this study revealed that KA-induced behavioural changes as early as latent phase after SE. This provides an insight into relationship between behavioural alteration and duration of epileptic state. All these findings might be useful for future researches, which looking into possible neuroprotective pathways of curcumin involved in epileptogenesis.

\section{Conflict of interest:}

The author (s) declares no conflict of interest.

\section{Financial support and sponsorship:}

This study was supported under a grant from Ministry of Higher Education Malaysia (grant no.: FRGS/1/2012/ SKK02/UKM/02/2).

\section{REFERENCES}

1. Tellez-Zenteno JF, Patten SB, Jette N, Williams J, Wiebe S. Psychiatric comorbidity in epilepsy: A population-based analysis. Epilepsia 2007;48:2336-44.

2. Taylor J, Kolamunnage-Dona R, Marson AG, Smith PE, Aldenkamp AP, Baker GA. Patients with epilepsy: Cognitively compromised before the start of antiepileptic drug treatment? Epilepsia 2010;51:48-56.

3. Groticke I, Hoffmann K, Loscher W. Behavioural alterations in the pilocarpine model of temporal lobe epilepsy in mice. Exp Neurol 2007;207:329-39.

4. Groticke I, Hoffmann K, Loscher W. Behavioural alterations in a mouse model of temporal lobe epilepsy induced by intrahippocampal injection of kainate. Exp Neurol 2008;213:71-83.

5. Brooks-Kayal AR, Bath KG, Berg AT, Galanopoulou AS, Holmes GL, Jensen FE, et al. Issues related to symptomatic and disease-modifying treatments affecting cognitive and neuropsychiatric comorbidities of epilepsy. Epilepsia 2013;54:44-60.

6. Ben-Ari Y, Tremblay E, Riche D, Ghilini G, Naquet R. Electrographic, clinical and pathological alterations following systemic administration of kainic acid, bicuculline or pentetetrazole: Metabolic mapping using the deoxyglucose method with special reference to the pathology of epilepsy. Neuroscience 1981;6:1361-91.

7. Covolan L, Mello LE. Temporal profile of neuronal injury following pilocarpine or kainic acid-induced status epilepticus. Epilepsy Res 2000;39:133-52.

8. Yow HY, Ahmad N, Makmor Bakry M. Pathogenesis of Epilepsy: Challenges in Animal Models. Iran J Basic Med Sci 2013;16:1119-32.

9. Levesque M, Avoli M. The kainic acid model of temporal lobe epilepsy. Neurosci Biobehav Rev 2013;37:2887-99.

10. Noor NA, Aboul Ezz HS, Faraag AR, Khadrawy YA. Evaluation of the antiepileptic effect of curcumin and Nigella sativa oil in the pilocarpine model of epilepsy in comparison with valproate. Epilepsy Behav 2012;24:199-206.

11. Sharma V, Nehru B, Munshi A, Sharma S, Khanna P. Protective effect of curcumin in behavioral impairment induced by pentylenetetrazol in rats. J Pharmacy Res 2011;4:11-4.

12. Jyoti A, Sethi P, Sharma D. Curcumin protects against electrobehavioral progression of seizures in the iron-induced experimental model of epileptogenesis. Epilepsy Behav 2009;14:300-8.

13. Mehla J, Reeta KH, Gupta P, Gupta YK. Protective effect of curcumin against seizures and cognitive impairment in a pentylenetetrazole-kindled epileptic rat model. Life Sci 2010;87:596-603.

14. Choudhary KM, Mishra A, Poroikov VV, Goel RK. Ameliorative effect of curcumin on seizure severity, depression like behaviour, learning and memory deficit in post-pentylenetetrazole-kindled mice. Eur J Pharmacol 2013;704:33-40.

15. Racine RJ. Modification of seizure activity by electrical stimulation. II. Motor seizure. Electroencephalogr Clin Neurophysiol 1972;32:281-94.

16. Du P, Tang HY, Li X, Lin HJ, Peng WF, Ma Y, et al. Anticonvulsive and antioxidant effects of curcumin on pilocarpine-induced seizures in rats. Chin Med J 2012;125:1975-9.

17. Gupta YK, Briyal S, Sharma M. Protective effect of curcumin against kainic acid induced seizures and oxidative stress in rats. Indian J Physiol Pharmacol 2009;53:39-46.

18. Gu J, Lynch BA, Anderson D, Klitgaard H, Lu S, Elashoff $\mathrm{M}$, et al. The antiepileptic drug levetiracetam selectively modifies kindling-induced alterations in gene expression in the temporal lobe of rats. Eur J Neurosci 2004;19:334-45.

19. Ramos A, Berton O, Mormede P, Chaouloff F. A multiple-test study of anxiety-related behaviours in six inbred rat strains. Behav Brain Res 1997;85:57-69.

20. Ennaceur A, Delacour J. A new one-trial test for neurobiological studies of memory in rats. 1: Behavioral data. Behav Brain Res 1988;31:47-59.

21. Ohl F. Testing for anxiety. Clin Neurosci Res 2003;3:233-8.

22. Antunes M, Biala G. The novel object recognition memory: Neurobiology, test procedure and its modifications. Cogn Process 2012;13:93-110.

23. Sharma S, Rakoczy S, Brown-Borg H. Assessment of spatial memory in mice. Life Sci 2010;87:521-36.

24. Pearson JN, Schulz KM, Patel M. Specific alterations in the performance of learning and memory tasks in models of chemoconvulsant-induced status epilepticus. Epilepsy Res 2014;108:1032-40.

25. Kwan P, Brodie MJ. Drug treatment of epilepsy: When does it fail and how to optimize its use? CNS Spectr 2004:9:110-19.

26. Lamberty Y, Falter U, Gower AJ, Klitgaard H. Anxiolytic profile of the antiepileptic drug levetiracetam in the Vogel conflict test in the rat. Eur J Pharmacol 2003;469:97-102.

27. Mazza M, Martini A, Scoppetta M, Mazza S. Effect of levetiracetam on depression and anxiety in adult epileptic patients. Prog Neuropsychopharmacol Biol Psychiatry 2008;32:539-43.

28. Campos AC, Fogaca MV, Aguiar DC, Guimaraes FS. Animal models of anxiety disorders and stress. Rev Bras Psiquiatr 2013;35:S101-S11.

29. Cavas M, Beltran D, Navarro JF. Behavioural effects of dimethyl sulfoxide (DMSO): changes in sleep architecture in rats. Toxicol Lett 2005;157:221-32.

30. Castro CA, Hogan JB, Benson KA, Shehata CW, Landauer 


\section{www.ijpsonline.com}

MR. Behavioural effects of vehicle: DMSO, ethanol, Tween-20, Tween-80 and emulphor-620. Pharmacol Biochem Behav 1995;50:521-6.

31. Ramos A. Animal models of anxiety: do I need multiple tests? Trends Pharmacol Sci 2008;29:493-8.

32. Kubova H, Mares P, Suchomelova L, Brozek G, Druga R, Pitkanen A. A Status epilepticus in immature rats leads to behavioural and cognitive impairment and epileptogenesis. Eur J Neurosci 2004;19:3255-65.

33. Maia GH, Quesado JL, Soares JI, do Carmo JM, Andrade PA, Andrade JP, et al. Loss of hippocampal neurons after kainate treatment correlates with behavioural deficits. PLoS One 2014;9:1-11.

34. Detour J, Schroeder H, Desor D, Nehlig A. A 5-month period of epilepsy impairs spatial memory, decreases anxiety, but spares object recognition in the lithium-pilocarpine model in adult rats. Epilepsia 2005;46:499-508.

35. Williams PA, White AM, Clark S, Ferraro DJ, Swiercz W, Staley KJ, et al. Development of spontaneous recurrent seizures after kainate-induced status epilepticus. J Neurosci 2009;29:2103-12.

36. Cavarsan CF, Queiroz CM, Dos Santos JG, Xavier GF, Mello LE, Covolan L. Reduced hippocampal dentate cell proliferation and impaired spatial memory performance in aged-epileptic rats. Front Neurol 2013;4:106.

37. Walsh RN, Cummins RA. The open-field test: a critical review. Psychol Bull 1976;83:482-504.

38. Ben-Ari Y. Limbic seizure and brain damage produced by kainic acid: Mechanisms and relevance to human temporal lobe epilepsy. Neuroscience 1985;14:375-403.

39. Deacon RM, Rawlins JN. Hippocampal lesions, species- typical behaviours and anxiety in mice. Behav Brain Res 2005;156:241-9.

40. Bannerman DM, Deacon RM, Offen S, Friswell J, Grubb M, Rawlins JN. A double dissociation of function within the hippocampus: Spatial memory and hyponeophagia. Behav Neurosci 2002;116:884-901.

41. Huang CW, Pai MC, Tsai JJ. Comparative cognitive effects of levetiracetam and topiramate in intractable epilepsy. Psychiatry Clin Neurosci 2008;62:548-53.

42. Lamberty Y, Margineanu DG, Klitgaard H. Absence of negative impact of levetiracetam on cognitive function and memory in normal and amygdala-kindled rats. Epilepsy Behav 2000;1:333-42.

43. Koo DL, Hwang KJ, Kim D, Kim YJ, Kim JY, Shin W, et al. Effects of levetiracetam monotherapy on the cognitive function of epilepsy patients. Eur Neurol 2013;70:88-94.

44. Sanchez PE, Zhu L, Verret L, Vossel KA, Orr AG, Cirrito JR, et al. Levetiracetam suppresses neuronal network dysfunction and reverses synaptic and cognitive deficits in an Alzheimer's disease model. Proc Natl Acad Sci USA 2012;109:2895-3.

45. Zhou JL, Zhao Q, Holmes GL. Effect of levetiracetam on visual-spatial memory following status epilepticus. Epilepsy Res 2007;73:65-74.

46. Ahmad M. Protective effects of curcumin against lithiumpilocarpine induced status epilepticus, cognitive dysfunction and oxidative stress in young rats. Saudi J Biol Sci 2013;20:155-62.

47. Sutcliffe JS, Marshall KM, Neill JC. Influence of gender on working and spatial memory in the novel object recognition task in the rat. Behav Brain Res 2007;177:117-52. 